\title{
Relación de Autorregulación del Aprendizaje y Autoeficacia General con las Metas Académicas de Estudiantes Universitarios
}

Carmen G. Covarrubias-Apablaza(1), Hedy Acosta-Antognoni(2) y Michelle Mendoza-Lira( ${ }^{(3)}$

(1) Vicerrectoría Académica, Universidad Central de Chile, La Serena, Chile. (e-mail: carmen.covarrubias@ucentral.cl)

(2) Facultad de Psicología, Universidad de Talca, Talca, Chile (e-mail: hacosta@utalca.cl)

(3) Facultad de Educación y Ciencias Sociales, Universidad Andres Bello, Viña del Mar, Chile

(e-mail: michelle.mendoza@unab.cl)

Recibido Mar. 22, 2019; Aceptado May. 18, 2019; Versión final Jul. 8, 2019, Publicado Dic. 2019

\begin{abstract}
Resumen
Basado en la Teoría Social Cognitiva y las Teorías del Aprendizaje, se analiza la relación entre autorregulación del aprendizaje y autoeficacia general en las dimensiones de las metas académicas de estudiantes universitarios. La muestra está compuesta por 231 participantes pertenecientes a una Universidad Pública Chilena, cuyas carreras están asociadas a las ciencias de economía, ingeniería, salud, ciencias sociales y humanidades. Los participantes cumplimentaron tres instrumentos de autoinforme con una duración aproximada de 15 minutos. Se probaron tres modelos a través de análisis de regresión lineal con el paquete estadístico SPSS (v. 22.0). Los resultados muestran que el Modelo 1 ( $\mathrm{M}_{1=\text { Autorregulación del }}$ aprendizaje y Autoeficacia general a Metas de aprendizaje) es el que presenta mejores indicadores para explicar las metas académicas en su subdimensión metas de aprendizaje, con una varianza explicada de $43 \%$. Se concluye que la Universidad debe potenciar acciones que apoyen a los estudiantes universitarios para diseñar sus metas de aprendizaje.
\end{abstract}

\section{Self-regulation Learning and General Self-efficacy and their relation with Academic Goals in University Students}

\begin{abstract}
Based on Social Cognitive Theory and Learning Theories, the aim of the study is to analyze the relationship between learning self-regulation and self-efficacy beliefs in the dimensions of the academic goals of university students. The sample is composed of 231 participants belonging to a Chilean Public University, whose careers are associated with the sciences of economics, engineering, health, social sciences and humanities. The participants completed three self-report instruments with an approximate timing of 15 minutes. Three models were tested through linear regression analysis with the statistical package SPSS (v. 22.0). The results show that Model 1 ( $\mathrm{M}_{1}$ = Self-regulation learning and General self-efficacy to Academic goals) presents the best indicators to explain the academic goals in its sub-dimension learning goals, with an explained variance of $43 \%$. It is concluded that the University must promote actions that support university students for designing their learning goals.
\end{abstract}




\section{INTRODUCCIÓN}

Las instituciones de educación superior deambulan en un escenario dinámico y complejo, más aún cuando tienen la responsabilidad de generar y promover el capital humano y cultural de una nación. Dado lo anterior, la heterogeneidad de los estudiantes universitarios que acceden a Universidades en Chile es una realidad desde hace tres décadas. Esto ha promovido transformaciones institucionales, curriculares y académicas, a tal punto, que las miradas hacia sus modelos educativos se han vuelto prácticas sistemáticas de mejora continua para asegurar la calidad. Lo anterior tiene un importante trasfondo, porque los futuros egresados además de ser competentes en sus áreas de especialidad- deben desenvolverse en una sociedad global. En consecuencia, las universidades poseen la oportunidad de formar profesionales integrales con recursos personales cognitivos, procedimentales, actitudinales y, por qué no decirlo, valóricos, para responder a las demandas de una sociedad en constante cambio (Agudo et al., 2013). Este escenario implica desarrollar conocimientos propios de una disciplina y promover la competencia de aprender lo largo de la vida (Bandura, 2012). Esto representa, sin duda, una tarea compleja, dado que los estudiantes deben movilizar sus recursos personales para alcanzar los objetivos impuestos por ellos y por la institución.

Estos recursos personales, entendidos como autoevaluaciones positivas referidas a la percepción de las capacidades personales para controlar e influir en el entorno, regulan las dimensiones afectivas, cognitivas y conductuales de los estudiantes universitarios para el logro académico (Bakker y Demerouti, 2013; BenEliyahu y Linnenbrink-García, 2015). Dicho de otra manera, los estudiantes que se esfuerzan, persisten, planean, focalizan la atención y, además, procesan la información y utilizan estrategias de aprendizaje tendrían mayores posibilidades de alcanzar los objetivos personales e institucionales (Torrano y Soria, 2017). No obstante, las instituciones de educación superior, no siendo responsables de las decisiones que toman los estudiantes, sí tienen la responsabilidad de ofrecer espacios académicos y oportunidades de aprendizaje que vayan más allá de resolver problemas, elaborar informes o responder una prueba; esto es que los estudiantes reflexionen sobre qué, cómo y por qué aprenden (Ellis et al., 2014). Más aún, los egresados de universidades logran una mayor empleabilidad cuando movilizan competencias para adaptarse a las necesidades del mundo laboral como el aprendizaje autónomo y permanente, la toma de decisiones, la retroalimentación del desempeño personal y colectivo, la creatividad, el pensamiento crítico, trabajo en equipo, la resolución de problemas, entre otras.

Considerando que las universidades han adoptado un modelo educativo basado en competencias, el cual trajo consigo cambios en el quehacer institucional, curricular y académico, esta investigación busca relevar qué factores resultan determinantes en el aprendizaje de los estudiantes universitarios. Para ello, el aprendizaje autorregulado, la autoeficacia general y las metas académicas constituyen constructos teóricos asociados a procesos, cognitivos, motivacionales y conductuales que han focalizado numerosos esfuerzos, desde la psicología educativa, por estudiarlos (Torrano y Soria, 2017). En razón de lo anterior, producto de una profunda reflexión, y bajo la aproximación de la Teoría Social Cognitiva (Bandura, 2012) y las Teorías del Aprendizaje (Zimmerman, 2013), es relevante evaluar el rol de la autoeficacia, la autorregulación del aprendizaje y las metas académicas en estudiantes universitarios. Dicho de otro modo, los estudiantes universitarios, además de juzgarse capaces, deben autorregular su aprendizaje, orientados por metas académicas profundas y autónomas.

\section{AUTORREGULACIÓN DEL APRENDIZAJE}

La autorregulación del aprendizaje alberga una considerable influencia en los procesos de enseñanzaaprendizaje y, como consecuencia, es una de las áreas de estudio más importante de la psicología educativa (Panadero, 2017). Conceptualmente, la autorregulación constituye un proceso que activa y mantiene los pensamientos, las conductas y las emociones para la consecución de las metas que se han propuesto los individuos (Zimmerman, 2013). Otros autores indican que la autorregulación es un proceso de control ejercido por los sujetos en sus pensamientos, acciones, emociones y motivaciones mediante estrategias que los dirijan hacia los objetivos propuestos (Panadero y Alonso-Tapia, 2014). Por su parte, Bandura (2012) sostiene que cuando los estudiantes autorregulan su aprendizaje propician el establecimiento de metas, movilizando los esfuerzos y recursos personales con tal de conseguirlas. Los estudiantes universitarios que autorregulan su aprendizaje cuentan no sólo con la capacidad, sino que también con la voluntad de aprender y, para ello, los procesos que autorregulan su aprendizaje resultan cruciales para incrementar la motivación y el aprendizaje académico (Rosário et al., 2007). Por otro lado, Panadero (2017) ha evidenciado que la autorregulación contempla el control sobre pensamientos, acciones y emociones, dirigido a la consecución de los objetivos, los que no necesariamente tienen orientación al aprendizaje. En consonancia con lo anterior, Ben-Eliyahu y Linnenbrink-García (2015) han revelado que la autorregulación del aprendizaje es utilizada deliberadamente por los estudiantes en los cursos favoritos, mientras que en los cursos menos favoritos la inversión de energía y la activación de la autorregulación es menor. Si bien, lo anterior parece obvio, los autores también han hecho hincapié que un estudiante autorregulado y efectivo en la utilización de sus 
estrategias de autorregulación gestiona tres niveles de aprendizaje: lo cognitivo (p.e., la concentración), lo conductual (p.e., planificación estratégica) y lo emocional (p.e., ansiedad).

De acuerdo al modelo teórico cíclico de fases de Zimmerman (2013), ampliamente aceptado (Lehmann et al., 2014) y explicado, recientemente, por Panadero y Alonso-Tapia (2014), la autorregulación del aprendizaje en los estudiantes está compuesta por tres fases: (1) planificación, (2) ejecución y (3) autorreflexión. En la primera fase, los estudiantes analizan la tarea a realizar en cuanto a sus características y valor, condicionando la motivación y el esfuerzo, así como los objetivos y el plan de acción para conseguirlos. Cabe destacar que en esta primera fase las creencias automotivadoras, como la autoeficacia, las expectativas de resultado, el valor de la tarea, el interés y la orientación a metas, juegan un rol clave. Por su parte, la segunda fase implica el despliegue de acciones o procesos para realizar lo que han planificado previamente. Durante esta fase, los estudiantes no sólo tienen que concentrarse, sino, además, seleccionar las mejores estrategias de aprendizaje, manteniendo el interés y motivación por alcanzar los objetivos impuestos en la etapa anterior. La auto-observación con la auto-monitorización y el auto-registro, así como el autocontrol metacognitivo y motivacional constituyen procesos indispensables para activar la fase. Finalmente, durante la tercera fase, el auto-juicio y la auto-rreacción de los estudiantes serán determinantes para valorar el trabajo, explicar las razones y causas de sus éxitos o errores y asociar emociones. De ahí que la autoevaluación, la atribución causal, la autosatisfacción e inferencia adaptativa resultan fuentes de una evaluación positiva o negativa final del proceso de aprendizaje. Dado lo anterior, la autorregulación del aprendizaje constituye un sinfín de procesos de carácter cognitivos, metacognitivos y motivacionales que los estudiantes activan ante las eventualidades académicas impuestas por la universidad.
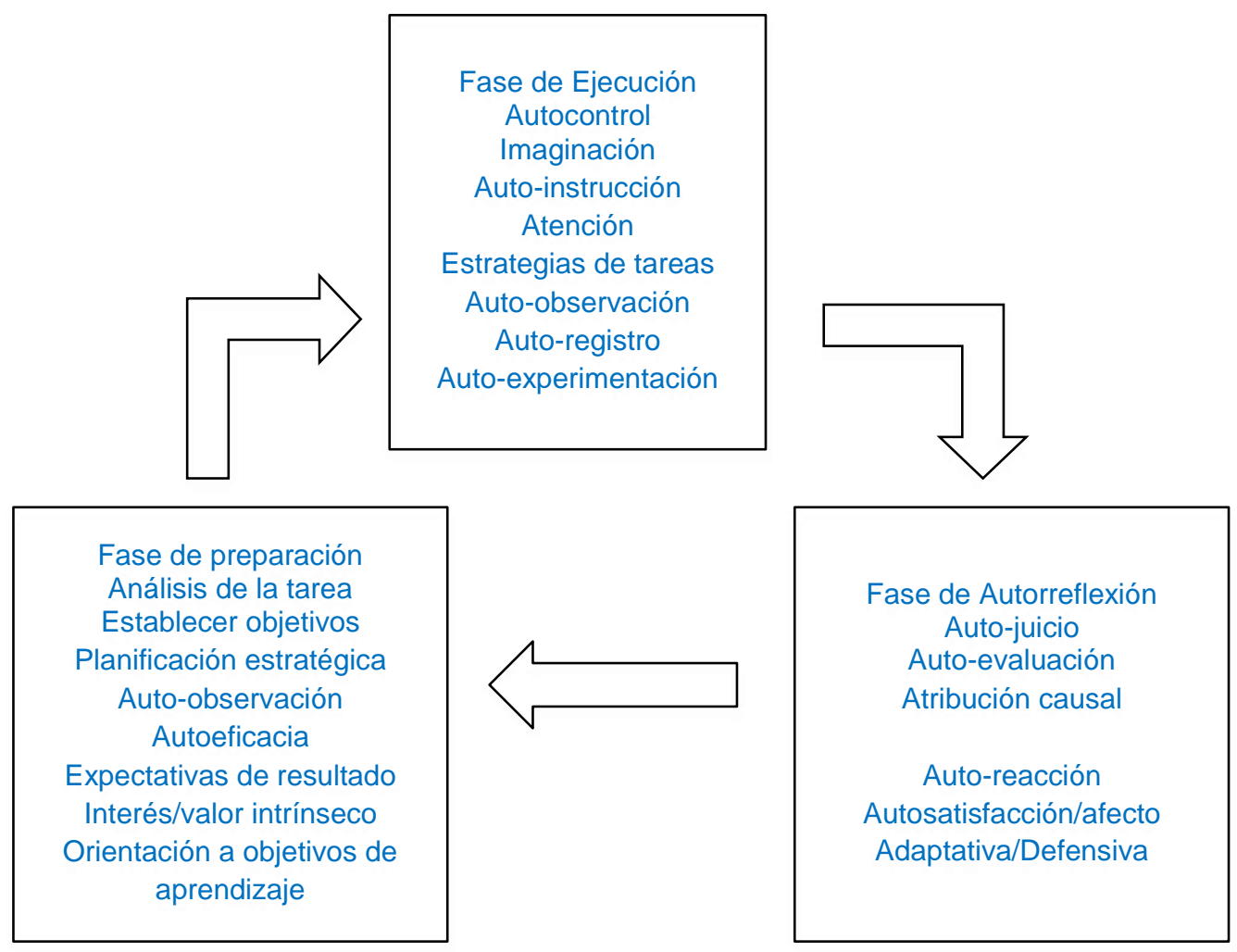

Fig. 1: Fases y procesos de la autorregulación (Adaptada de Zimmerman, 2013, p. 142)

Cabe destacar que los estudios de autorregulación del aprendizaje han sido bastante prolíficos. Ellis et al. (2014), en una revisión de la literatura, concluyeron que los procesos de enseñanza-aprendizaje que tributan significativamente en los rendimientos académicos de los estudiantes están basados en el uso de estrategias metacognitivas explícitas, siendo el modelado la estrategia más utilizada por los profesores. Un estudio sobre entrenamiento en estrategias de autorregulación del aprendizaje ha favorecido de manera significativa tanto el conocimiento de dichas estrategias como la autoeficacia en el uso de las mismas en estudiantes universitarios (Cerezo et al., 2018). Interesante, también, es la investigación cuasi-experimental sobre autorregulación del aprendizaje en entornos hipermedia y la influencia de los conocimientos previos en estudiantes universitarios de Taub et al. (2014), quienes demostraron que, independientemente del nivel bajo o alto de conocimientos previos, los estudiantes participantes de la investigación utilizaron estrategias cognitivas y metacognitivas. Por su parte, considerando el avance de la educación en línea Lehmann et al, (2014) consideran que la autorregulación del aprendizaje constituye un punto crítico para el desempeño 
eficiente de los estudiantes universitarios, así como también la metacognición lo es para las tareas de escritura. Por ejemplo, las evaluaciones con mapas conceptuales exitosas no sólo implican interés, esfuerzo y comprensión, sino que además dominio de la materia en sus aspectos estructurales y semánticos. Para finalizar, con perspectiva de género Torrano y Soria (2017) han discutido que las estudiantes pese a manifestar menores niveles de autoeficacia en matemáticas, utilizan mayormente estrategias de aprendizaje lo que podría estar explicado por la modestia al contestar, así como también por una evaluación más realista de sus fortalezas y debilidades.

\section{AUTOEFICACIA}

Para Bandura (2012), la autoeficacia es un juicio de capacidad que poseen las personas en torno a los desempeños demandados por los contextos donde se desenvuelven; al igual que la autorregulación del aprendizaje, no constituye un rasgo o una característica personal. Si bien las habilidades o las competencias son relevantes para las creencias de autoeficacia, mayor aún lo son lo que las personas pueden hacer con ellas. Ahora bien, fuertes o débiles creencias de eficacia generan impactos diferenciados en los pensamientos, afectos y conductas de las personas en un amplio y, al mismo tiempo, estable rango de situaciones y contextos. Cabe destacar que las creencias de eficacia constituyen la clave de estudiantes autodirigidos y con un óptimo funcionamiento intelectual en los contextos académicos. En consonancia con lo anterior, para Linnenbrink y Pintrich (2003), la autoeficacia resulta indispensable para la mantención del compromiso y el aprendizaje en los estudiantes. De tal manera, los estudiantes universitarios reciben una influencia moderadora de las creencias de eficacia que ellos tienen (Honicke y Broadbent, 2016). Tal es así, que los estudiantes con altos niveles de autoeficacia poseen mayores posibilidades de obtener un mejor rendimiento académico de aquellos que no lo tienen (Krumrei-Mancuso et al., 2013). Más aún, aquellos estudiantes que tienen una férrea confianza en sus capacidades no sólo seleccionan desafíos académicos más retadores, sino que además persisten ante las dificultades y adaptan estrategias de aprendizaje para enfrentar los fracasos (Mega et al., 2014). Por lo tanto, la autoeficacia es un predictor significativo de las autoatribuciones a la capacidad y el esfuerzo (Bandura, 2012).

Dado lo anterior, la autoeficacia de los estudiantes universitarios influye en la motivación por aprender, en las respuestas afectivas involucradas en sus desempeños y, como consecuencia, en el éxito académico. Por tanto, se puede entender que el aprendizaje de los estudiantes universitarios se ve influenciado por creencias de autoeficacia que activan mecanismos cognitivos, conductuales y motivacionales, con el propósito de realizar una tarea y alcanzar las metas (Usher y Pajares, 2008). También es relevante mencionar que las creencias de autoeficacia académica en estudiantes fomenta la toma de decisiones para resolver los problemas o las tareas demandadas por el contexto universitario (Pajares, 2003). Dada la naturaleza recíproca de la autoeficacia entre persona, conducta y ambiente (Beatson et al, 2018), los estudiantes que tienen altos niveles de autoeficacia se caracterizan por: a) ver los problemas como desafíos y no como amenazas; b) mantener el esfuerzo y compromiso por lo objetivos planteados; c) retroalimentar sus desempeños; d) responsabilizar a sus fracasos por el escaso esfuerzo y no por la capacidad; e) optar por tareas más desafiantes; f) gestionar procesos de autorregulación; g) establecer metas (Komarraju y Nadler, 2013; Linnenbrink y Pintrich, 2003; Pajares 2003; Wolters, 2004).

Por otro lado, diversos estudios han evidenciado que las creencias de autoeficacia pueden verse nutridas por las experiencias directas (Beatson et al., 2018), la persuasión verbal (Ahn et al., 2016), el aprendizaje vicario (Byrne et al., 2014). En consonancia con lo anterior, Yurt (2014) ha revelado que las fuentes de autoeficacia como las experiencias personales, las persuasiones sociales y los estados fisiológicos constituyen predictores significativos en el rendimiento de matemáticas en estudiantes de secundaria. También, las creencias de autoeficacia reciben influencia del apoyo familiar (Weiser y Riggio, 2010) y de las emociones negativas (Villavicencio y Bernardo, 2013).

\section{METAS ACADÉMICAS}

Las metas constituyen los propósitos que albergan las personas para iniciar y desarrollar comportamientos orientados hacia un logro. También constituyen representaciones cognitivas y motivadoras de las conductas futuras que ejecutan las personas en los contextos donde se desenvuelven. No obstante, cabe señalar que la orientación de las metas, como patrón integrado de creencias, marca la diferencia en por qué aprenden o estudian los estudiantes (Pintrich, 2000). Para Dweck (1986), la implicación de los estudiantes en sus quehaceres se explicaba por dos razones: (1) estudiar para aumentar la competencia y dominar algo nuevo; o (2) estudiar para no parecer incompetente frente a otros y él mismo. Mientras en la primera orientación, metas de aprendizaje, el foco de los estudiantes está en la satisfacción por el esfuerzo realizado, en las metas de desempeño lo que ellos valoran es la demostración de las habilidades que presentan (Linnenbrink, 2005). Dicho de otra manera, los estudiantes orientados a aprender y, como resultado, a mejorar sus capacidades y aumentar sus conocimientos seleccionarán actividades académicas más desafiantes, enfrentarán los 
obstáculos, aumentarán esfuerzos y seleccionarán las estrategias más efectivas, independientemente de los éxitos o fracasos de sus ejecuciones. Por el contrario, los estudiantes orientados por el desempeño buscarán actividades que resguarden su seguridad personal. Esta estrategia defensiva disminuye sus esfuerzos para enfrentar retos y, en caso de obtener resultados desfavorables, lo asocian a sus capacidades (Pulkka y Niemivirta 2013; Pintrich, 2000).

A pesar de lo antes dicho, la teoría de los objetivos de logro se ha enriquecido bastante durante 25 años, logrando ciertos acuerdos en cuanto a que las metas de desempeño no siempre serían nocivas, y que podrían ser tan beneficiosas como las de dominio para la utilización de estrategias de aprendizaje y para aumentar los niveles de autoeficacia (Senko et al., 2011). Ahora bien, los hallazgos más recientes incorporan los enfoques de acercamiento o evitación a la clásica mirada bidimensional de las metas: dominio y rendimiento. Dicho de otra manera, un estudiante puede tener una meta con orientación hacia el dominio y con un enfoque de acercamiento, disponiéndose hacia el dominio de aprender, comprender y mejorar, o hacia un enfoque de evitación donde primará el evitar las equivocaciones o la falta de dominio. Por el contrario, los estudiantes con una orientación hacia el rendimiento se adscribirán hacia un enfoque de acercamiento cuando deseen mostrarse superiores y mejores, o de evitación cuando no quieran hacer frente al fracaso o parecer incompetentes. Cabe señalar que Valle et al, (2006) han revelado una relación positiva entre el enfoque de acercamiento de las metas de rendimiento, el procesamiento estratégico de la información y la autorregulación del aprendizaje. Más aún reafirman lo anterior considerando el estudio de Wolters (2004) quien manifiesta que mostrarse eficaz ante otros podría activar la utilización de mecanismos autorreguladores para la mejora del aprendizaje.

Basándose en el modelo de Dweck (1986), Hayamizu et al. (1989) establecieron una tipología de metas: (1) de aprendizaje, (2) de logro y (3) de refuerzo social, de tal manera que la primera se asociaba a las metas de mejora de la competencia, mientras que la segunda y tercera se relacionaban con metas de rendimiento (Inglés et al. 2016). Con respecto a las metas de aprendizaje es importante indicar que orientan los comportamientos de los estudiantes hacia el desarrollo de las propias competencias; dicho de otra manera, se ven movilizados por el acto de aprender y, como consecuencia, de mejorar sus conocimientos y habilidades. Por el contrario, los estudiantes con una orientación de meta hacia los resultados, movilizan competencias y estrategias académicas para alcanzar buenos resultados académicos. Por último, las metas de refuerzo social están centradas en la obtención del reconocimiento o aprobación de pares, profesores y familia. Además, cabe señalar que las metas académicas no son excluyentes, ya que ante tareas los estudiantes pueden estar dirigidos por una u otra según el contexto, las características personales o los desafíos impuestos por una actividad en particular (Dull et al., 2015; Gámez et al., 2014).

Sumado a ello, los perfiles motivacionales con predominio de metas de aprendizaje están asociados a mejores desempeños con un rol clave de la autoeficacia (Valle et al. 2015). Incluso, el autoconcepto en estudiantes universitarios influyó significativamente en la elección de metas y en las necesidades de autonomía, competencia y vinculación (Gámez et al., 2014). Por su parte, Dull et al. (2015) - en un estudio con universitarios que cursaban la carrera de contabilidad - revelaron que la orientación hacia una o varias metas tenía un impacto positivo en las calificaciones y en la autoeficacia. Desde una mirada longitudinal, Pulkka y Niemivirta (2013) han manifestado la estabilidad que presentan las orientaciones de metas en los estudiantes y, aun cuando haya un cambio de orientación, éste será hacia una similar (p.e., de aprendizaje a éxito). Incluso, los estudiantes con foco en el aprendizaje valoran positivamente las experiencias personales de aprendizaje y el esfuerzo.

\section{METODOLOGÍA}

La metodología explica, entre otros aspectos, los objetivos de la investigación, la población, la muestra, las hipótesis y el procesamiento de los datos.

\section{Objetivos}

El objetivo de este estudio es analizar la relación entre autorregulación del aprendizaje y autoeficacia general en las dimensiones (i.e., metas de aprendizaje, metas de logro y metas de refuerzo social) de las metas académicas de estudiantes universitarios. Con el propósito de otorgar respuesta al objetivo planteado, el estudio sigue una metodología cuantitativa no experimental, de carácter transversal y tipo correlacional para comprobar las hipótesis y explorar las relaciones existentes entre los constructos mencionados. 


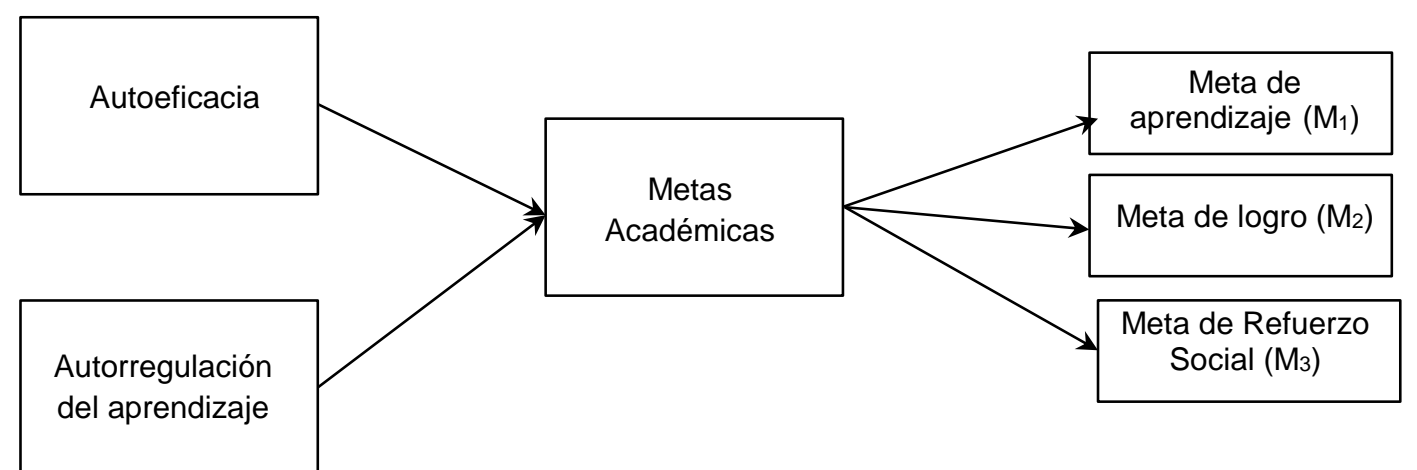

Fig. 2: Modelo de Investigación

\section{Población y Muestra}

La muestra no probabilística y por conveniencia está compuesta por 231 estudiantes universitarios de segundo año de una Universidad Pública Chilena, de los cuales $157(68 \%)$ eran mujeres y $74(32 \%)$ eran hombres con edades comprendidas entre 18 y 29 años $(M=19.8$ y $\mathrm{DT}=1.39)$. Cabe señalar que los participantes pertenecen a carreras asociadas a las ciencias de Ingeniería, Salud, Sociales y Humanidades, con el propósito de abarcar las diversas disciplinas que oferta la institución de educación superior.

Tabla 1: Características de la muestra

\begin{tabular}{|c|c|c|}
\hline \multicolumn{2}{|c|}{ Variables } & $\%$ \\
\hline \multirow{3}{*}{ Sexo } & Mujer & 68 \\
\cline { 2 - 3 } & Hombre & 32 \\
\hline \multirow{3}{*}{$\begin{array}{c}\text { Edad } \\
\text { (años) }\end{array}$} & 18 a 19 & 49.8 \\
\cline { 2 - 3 } & 20 a 21 & 40.7 \\
\cline { 2 - 3 } & 22 a 29 & 9.6 \\
\hline \multirow{4}{*}{ Área Carrera } & Ingeniería & 35 \\
\cline { 2 - 3 } & Salud & 34.3 \\
\cline { 2 - 3 } & Sociales & 20.3 \\
\cline { 2 - 3 } & Humanidades & 10.4 \\
\hline
\end{tabular}

\section{Hipótesis}

Las hipótesis de este estudio son dos: $\mathrm{H}_{1}$ : Se espera que la autorregulación del aprendizaje y la autoeficacia general se relacionen positivamente con las dimensiones de las metas académicas (i.e., metas de aprendizaje, metas de logro y metas de refuerzo social) de estudiantes universitarios; y $\mathrm{H}_{2}$ : Se espera que la autoeficacia general tenga un peso relativo mayor en las dimensiones de las metas académicas (i.e., metas de aprendizaje, metas de logro y metas de refuerzo social) de estudiantes universitarios.

\section{Instrumentos}

Los instrumentos seleccionados para este estudio cumplen con el criterio de fiabilidad (valores iguales o mayores a .70) propuesto por Nunnally y Bernstein (1994): a) El Cuestionario de metas académicas de Hayamizu et al. (1989), adaptado por Hayamizu y Weiner (1991) y traducido y adaptado al español por Navas et al. (2002), explora los tipos de metas que orientan el quehacer académico de los estudiantes en 20 ítems distribuidos tres factores: a) metas de aprendizaje, compuesto por 8 ítems (p.e., "Estudio porque para mí es interesante resolver problemas"); b) metas de refuerzo social, compuesto por 6 ítems (p.e., "Estudio porque quiero ser elogiado por mis padres y profesores") y c) metas de logro, compuesto por 6 ítems (p.e., "Estudio porque quiero obtener buenas notas"). Los estudiantes responden por qué estudian en una escala Likert graduada de 1 (nunca) a 5 (siempre). El cuestionario presenta una consistencia interna de .85, distribuida en los tres factores del cuestionario. Las características psicométricas del instrumento arrojadas por la respuesta de 542 estudiantes universitarios chilenos, revelan que el instrumento tiene consistencia interna (.83) y validez de constructo donde los tres factores explican el $51 \%$ de la varianza total (Pérez et al., 2009); b) La Escala de autoeficacia general adaptada y validada al español por Baessler y Schwarzer (1996), compuesta por 10 ítems y una escala Likert de cuatro puntos de 1 (incorrecto) a 4 (cierto), evalúa la creencia 
de competencia personal para resolver tareas nuevas y afrontar las dificultades del contexto (p.e., "Puedo resolver problemas difíciles si me esfuerzo lo suficiente"). La fiabilidad de la escala es de .82. Cid et al. (2010), demuestran que el instrumento reúne las características psicométricas para aplicarse en muestra chilena. El coeficiente alfa de Cronbach fue de .84; y c) El Inventario de los procesos de autorregulación del aprendizaje (IPAA) de Rosário et al. 2007) recoge información sobre el uso de estrategias de autorregulación del aprendizaje, está conformado por 12 ítems distribuidos en las fases de: a) planificación, constituida por 4 ítems (p.e., "Hago un plan antes de comenzar a hacer un trabajo escrito. Pienso lo que voy a hacer y lo que necesito para conseguirlo"); b) ejecución, constituida por 4 ítems (p.e., "Busco un sitio tranquilo y donde pueda estar concentrado para estudiar") y c) evaluación, constituida por 4 ítems (p.e., "Comparo las notas que saco con los objetivos que había marcado para esa asignatura"). Dichos ítems son evaluados en una escala Likert de 5 puntos de 1 (nunca) a 5 (siempre) y en el cual los estudiantes manifiestan cómo aprenden en contextos académicos. Cabe destacar que la consistencia general de la escala es de .78, en sus tres dimensiones. La adaptación y validación en estudiantes universitarios chilenos arrojó que el IPAA es un cuestionario válido y fiable, con un alfa de .81 y una varianza total explicada de $58,8 \%$ para ser aplicado en dicho contexto (Bruna et al., 2017).

\section{Recogida y análisis de datos}

En primera instancia, se solicitó a las autoridades universitarias pertinentes la aplicación de los instrumentos en contexto de clase, quienes autorizaron y valoraron la iniciativa. Posteriormente, los estudiantes que accedieron a participar de manera voluntaria, cumplimentaron un consentimiento informado que garantizó la confidencialidad de la información y el cumplimiento de los aspectos éticos del estudio. Finalmente, los estudiantes respondieron tres cuestionarios de autoinforme en condiciones de aula, en el horario de clases habitual y con tiempo suficiente para responder los reactivos de los instrumentos. La duración aproximada de aplicación de los cuestionarios fue de 15 minutos. En cuanto al análisis de datos, en primer lugar, se realizaron análisis descriptivos (i.e., media, desviación típica). En segundo lugar, se evaluó la fiabilidad de las escalas del estudio a través del alfa de Cronbach ( $\alpha$ ), considerando el punto de corte de Nunnally y Bernstein (1994). En tercer lugar, se llevaron a cabo las intercorrelaciones (correlación de Pearson) de las escalas del estudio. En cuarto lugar, a través de análisis de regresión lineal, se probaron tres modelos: (1) $M_{1}=$ Autorregulación del aprendizaje y Autoeficacia general a Metas de aprendizaje; (2) $M_{2}=$ Autorregulación del aprendizaje y Autoeficacia general a Metas de logro y, (3) $M_{3}=$ Autorregulación del aprendizaje y Autoeficacia general a Metas de Refuerzo Social. Finalmente, se evaluaron los pesos relativos a través de los indicadores Beta $(\beta)$. Todos los análisis se llevaron a cabo con el paquete estadístico SPSS (22.0).

\section{RESULTADOS}

La Tabla 2 muestra los estadísticos descriptivos, la fiabilidad de las escalas y las intercorrelaciones. Como se puede apreciar, todas las escalas cumplen con el criterio propuesto por Nunnally y Bernstein (1994). Además, las intercorrelaciones de las escalas fueron positivas y significativas $(p<.001)$. La Tabla 3 muestra el Modelo $1\left(\mathrm{M}_{1}\right)$, donde Autoeficacia General y Autorregulación del Aprendizaje revelan una varianza explicada de las Metas de Aprendizaje de $\mathrm{R}^{2}=43 \%, \mathrm{~F}=87.73 ; p<.001$.

Tabla 2: Estadísticos descriptivos, fiabilidad $(\alpha)$ en diagonal e intercorrelaciones $(N=231) p<.001^{* * *}$

\begin{tabular}{|l|c|c|c|c|c|}
\hline \multicolumn{1}{|c|}{ Variables } & Media & $d t$ & 1 & 2 & 3 \\
\hline 1. Autoeficacia General & 3.23 & .41 & $(.82)$ & & \\
\hline 2. Metas Académicas & 3.47 & .54 & $.39^{\star * *}$ & $(.85)$ & \\
\hline 3. Autorregulación del Aprendizaje & 3.63 & .58 & $.43^{\star * *}$ & $.55^{\star * *}$ & $(.79)$ \\
\hline
\end{tabular}

Tabla 3: Modelo 1. Regresión Autoeficacia General y Autorregulación del aprendizaje a Metas de Aprendizaje ( $\mathrm{N}=231$ ) (Variable dependiente: Metas de Aprendizaje; $p<.001^{\star \star *}$ )

\begin{tabular}{|l|c|c|c|c|}
\hline Modelo & $\mathrm{R}^{2}$ & $\beta$ & $\mathrm{F}$ & $p$ \\
\hline $\mathrm{M}_{1}$ & .43 & & 87.73 & .00 \\
\hline Autoeficacia & & .28 & & .00 \\
\hline Autorregulación Aprendizaje & & .49 & & .00 \\
\hline
\end{tabular}

La Tabla 4 muestra el Modelo $2\left(\mathrm{M}_{2}\right)$, donde Autoeficacia General y Autorregulación del Aprendizaje revelan una varianza explicada de las Metas de Logro de $R^{2}=14 \%, F=91.37 ; p<.001$. En este modelo, los análisis reflejan que la autoeficacia general no es significativa en este modelo $(\beta=.0625 ; p=.36)$. 
Tabla 4: Modelo 2. Regresión Autoeficacia General y Autorregulación del aprendizaje a Metas de logro (Variable dependiente: Metas de Logro; $p<.001 ; p=n s^{\star \star \star}$ )

\begin{tabular}{|l|c|c|c|c|}
\hline Modelo & $\mathrm{R}^{2}$ & $\beta$ & $\mathrm{F}$ & $p$ \\
\hline $\mathrm{M}_{2}$ & .14 & & 20.04 & .00 \\
\hline Autoeficacia & & .06 & & .36 \\
\hline Autorregulación Aprendizaje & & .35 & & .00 \\
\hline
\end{tabular}

La Tabla 5 muestra el Modelo $3\left(\mathrm{M}_{3}\right)$, donde Autoeficacia General y Autorregulación del Aprendizaje evidencian ser no significativos, revelando una varianza explicada de las Metas de Refuerzo Social de $R^{2}=$ $1 \%, F=2.62 ; p<n s$.

Tabla 5: Modelo 3. Regresión Autoeficacia General y Autorregulación del aprendizaje a Metas de Refuerzo Social (Variable dependiente: Metas de Refuerzo Social; $p<.001 ; p=n s^{\star \star *}$ )

\begin{tabular}{|l|c|c|c|c|}
\hline Modelo & $\mathrm{R}^{2}$ & $\beta$ & $\mathrm{F}$ & $p$ \\
\hline $\mathrm{M}_{3}$ & .01 & & 2.62 & .08 \\
\hline Autoeficacia & & .03 & & .71 \\
\hline Autorregulación Aprendizaje & & .14 & & .06 \\
\hline
\end{tabular}

En cuanto a los pesos relativos, el indicador Beta $(\beta)$ señala que en los tres modelos la variable autorregulación del aprendizaje tiene un peso relativo mayor en cada una las dimensiones (i,e., metas de logro, metas aprendizaje y metas de refuerzo social) de las metas de aprendizaje ( $M_{1}: \beta=.49 ; M_{2}: \beta=.35 ; M_{3}$ : $\beta=.14)$.

\section{DISCUSIÓN}

El objetivo del estudio fue analizar la relación entre autorregulación del aprendizaje y autoeficacia en las dimensiones (i.e., metas de aprendizaje, metas de logro y metas de refuerzo social) de las metas académicas para una muestra de estudiantes universitarios chilenos. Los resultados indican que, de los tres modelos probados, el modelo más robusto es el $\mathrm{M}_{1}$, donde autorregulación del aprendizaje y autoeficacia general explican el $43 \%$ de la varianza explicada de la sub-dimensión metas de aprendizaje. Cabe señalar que, en coherencia con la Teoría Social Cognitiva (Bandura, 2012) y las Teorías del Aprendizaje (Zimmerman, 2013), para esta muestra de estudiantes universitarios, las correlaciones entre autorregulación del aprendizaje y autoeficacia general con las metas académicas han sido positivas y significativas, aunque sólo en la subdimensión metas de aprendizaje. Por lo tanto, si bien se confirman empíricamente las relaciones entre ellas, la H1 se comprobó sólo parcialmente.

Resulta interesante que el peso relativo mayor, en los tres modelos probados, recae en la autorregulación del aprendizaje, rechazándose la H2. Éste es un resultado que difiere con la Teoría Social Cognitiva, ya que ésta le otorga un papel influyente a la autoeficacia en los procesos motivacionales de los sujetos. Sin embargo, Sitzmann y Ely (2011), en un meta-análisis sobre el estado de investigación del aprendizaje autorregulado en un período de treinta años, mostraron que esta variable tenía los efectos más fuertes y positivos en el aprendizaje, las metas, la persistencia, el esfuerzo y la autoeficacia. No obstante, las discrepancias en los resultados de este estudio con otros, cuya primera hipótesis se comprueba parcialmente, mientras que la segunda se rechaza, podrían explicarse por dos razones. La primera, asociada a la utilización de autoinformes para la medición. Si bien son adecuados para aplicar a grandes muestras presentan dificultades para capturar el comportamiento real de los sujetos (Roth et al., 2016). De tal manera, los estudiantes universitarios responderían en función de lo esperado, sobreestimando sus percepciones en torno a las tres variables del estudio. La segunda, relacionada a una muestra no probabilística por conveniencia que ha capturado de manera heterogénea las relaciones en las percepciones de los estudiantes universitarios en las tres variables del estudio, lo que hace que los resultados no puedan ser ni generalizables ni tampoco concluyentes.

Con los matices propios de la investigación, este estudio complementa otros que han relacionado la autoeficacia y el uso de estrategias de aprendizaje autorregulado (Pool-Cibrián y Martínez-Guerrero, 2013), así como también las expectativas de autoeficacia, las metas académicas y su influencia en los perfiles motivacionales de estudiantes universitarios (Valle et al., 2015). Desde otra perspectiva, Al-Baddareen et al. (2015) partieron de la premisa de que la autoeficacia influiría en las metas que los sujetos seleccionan para aprender. Sin embargo, los resultados de esta investigación develaron que la autoeficacia no tuvo una correlación significativa con las metas de rendimiento. No obstante, constituye una realidad que muchos 
estudiantes universitarios carecen de conocimientos y competencias para monitorear y ajustar sus mecanismos de aprendizaje ante situaciones académicas demandantes o difíciles (Björk et al., 2013). Por lo mismo, y considerando que las estrategias de aprendizaje autorregulado pueden ser enseñadas, Rosário et al. (2015), mediante el programa de promoción de procesos y estrategias de aprendizaje, logró mejorar -entre varias variables motivacionales- la autoeficacia. Más aún, la incorporación de procesos de autoevaluación promueve el establecimiento y claridad en las metas, el monitoreo del aprendizaje y la reflexión de los resultados obtenidos; efectos significativos en la autorregulación del aprendizaje y la autoeficacia en los estudiantes universitarios (Panadero et al., 2017).

En esta misma línea, Cerezo et al. (2018) han evidenciado que el conocimiento de las estrategias de autorregulación del aprendizaje mejora significativamente la autoeficacia para el uso de dichas estrategias, siempre que se encuentren asociadas a metas de aprendizaje. Efectivamente, un estudiante autorregulado es un estudiante exitoso y, por lo tanto, sujeto activo de aprendizaje, donde la confianza en sus capacidades y la orientación de sus acciones lo dirigen hacia el logro de sus metas. Pese a ello, muchos estudiantes tienen serias dificultades para enfrentar eficazmente los desafíos académicos, siendo sujetos susceptibles de intervención o capacitación en variables motivacionales. Una intervención transcultural en Portugal, España, Chile y Mozambique logró promover el uso estrategias para el aprendizaje autorregulado y, además, mejorar la autoeficacia en estudiantes universitarios (Rosário et al., 2015). Lo anterior conduce a reconocer la importancia de que las instituciones de educación superior, además de promover en los estudiantes procesos de control sobre sus pensamientos, acciones, emociones y motivaciones, provean experiencias directas y reales, con el propósito de que sus aprendizajes se caractericen por la autonomía, profundidad y transferencia (Panadero, 2017; Zimmerman, 2013).

Por último señalar que las creencias de autoeficacia positivas constituyen un componente fundamental en la implicación de los estudiantes que, junto a procesos de autorregulación del aprendizaje, potencian las metas de mejora en la competencia personal. Todas ellas pueden ser trabajadas por la universidad como mecanismos efectivos con foco en el logro académico y en el desarrollo integral de los estudiantes universitarios (Beatson et al., 2018). En consideración de lo antes dicho, una evaluación de la relación entre autorregulación del aprendizaje y autoeficacia general con metas académicas otorga una mirada novedosa para explicar cómo aprenden o cómo enfrentan los estudiantes las demandas universitarias. Efectivamente, para un aprendizaje significativo y autónomo no basta sólo con "saberse los contenidos" de una asignatura particular, sino de disponer cuotas de iniciativa, confianza, esfuerzo y persistencia para lograr con éxito los desafíos académicos.

\section{CONCLUSIONES}

Del análisis y la discusión de los resultados de esta investigación se desprenden las siguientes conclusiones:

1) para esta muestra de estudiantes universitarios de una Universidad Pública Chilena, el peso relativo mayor en las metas de aprendizaje lo obtiene la autorregulación del aprendizaje, indicando que los estudiantes que gestionan sus tiempos de estudio, auto-observan sus conductas, buscan ayuda y orientan el logro académico hacia el aprendizaje más que el rendimiento;

2) como resultado novedoso, planificación, ejecución y evaluación de sus aprendizajes tendrían una mayor influencia que las creencias de eficacia;

3) estos resultados se deben analizar con cautela, debido a que la literatura le otorga a las creencias de eficacia un importante valor predictivo en los aprendizajes;

4) en este sentido, es relevante probar el modelo hipotetizado en esta investigación con una muestra probabilística más amplia y robusta que permita generalizar los resultados.

\section{REFERENCIAS}

Agudo, E., R. Hernández-Linares y otros dos autores, Competencias Transversales: Percepción de su desarrollo en el Grado en Ingeniería en Diseño Industrial y Desarrollo de Productos, doi: http://dx.doi.org/10.4067/S071850062013000500006, Formación Universitaria, 6(5), 39-50 (2013)

Ahn, H.S., M. Bong y S.I. Kim, Social Models in the Cognitive Appraisal of Self-efficacy Information, doi: https://doi.org/10.1016/j.cedpsych.2016.08.002, Contemporary Educational Psychology, 48, 149-166 (2017)

Al-Baddareen, G., S. Ghaith y M. Akour, Self-efficacy, Achievement Goals, and Metacognition as Predicators of Academic Motivation, doi: https://doi.org/10.1016/j.sbspro.2015.04.345, Procedia Social and Behavioral Science, 191, 2068-2073 (2015)

Baessler, J. y R. Schwarzer, Evaluación de la Autoeficacia: Adaptación Española de la Escala de Autoeficacia General, 
Ansiedad y Estrés, ISSN: 1134-7937, 2, 1-8 (1996)

Bakker, A.B. y E. Demerouti, La teoría de las demandas y los recursos laborales, doi: 10.5093/tr2013a16, Revista de Psicología del trabajo y las organizaciones, 29(3), 107-115 (2013)

Bandura, A., On the Functional Properties of Perceived Self-Efficacy Revisited, doi: 10.1177/0149206311410606, Journal of Management, 38(1), 9-44 (2012)

Beatson, N.J., D. Berg y J. Smith, The Impact of Mastery Feedback on Undegraduate Students' Self-efficacy Beliefs, doi: https://doi.org/10.1016/j.stueduc.2018.03.002, Studies in Educational Evaluation, 59, 58-66 (2018)

Ben-Eliyahu, A. y L. Linnenbrink-García, Integrating the regulations of affect, behavior, and cognition into self-regulated learning paradigms among secondary and post-secundary students, doi: 10.1007/s11409-014-9129-8, Metacognition Learning, 10, 15-42 (2015)

Björk, R., J. Dunlosky y N. Kornell, Self-regulated Learning: Beliefs, Techniques, and Illusions, doi: 10.1146/annurevpsych-113011-143823, Annual Review of Psychology, 64, 417-444 (2013)

Bruna, D., M.V. Pérez y otros dos autores, Propiedades Psicométricas del Inventario de Procesos de Autorregulación del Aprendizaje en Estudiantes Universitarios Chilenos, doi: https://doi.org/10.21865/RIDEP44.2.07, Revista Iberoamericana de Diagnóstico y Evaluación, 44(2), 77-91 (2017)

Byrne, M., B. Flood y J. Griffin, Measuring the Academic Self-efficacy of First-year Accounting Students, doi: https://doi.org/10.1080/09639284.2014.931240, Accounting Education, 23(5), 407-423 (2014)

Cerezo, R., E. Fernández y otros cuatro autores, El Papel Mediador de la Autoeficacia y la Utilidad entre el Conocimiento y el Uso de Estrategias de Autorregulación del Aprendizaje, doi: https://doi.org/10.1016/j.psicod.2018.08.001, Revista de Psicodidáctica, 24(1), 1-8 (2018)

Cid, P., A. Orellana y O. Barriga, Validación de la Escala de Autoeficacia General en Chile, doi: http://dx.doi.org/10.4067/S0034-98872010000500004, Revista Médica de Chile, 138(5), $551-557$ (2010)

Dull, R.B., L.L.F. Schleifer y J. McMillan, Achievement goal theory: The relationships of accounting students' goal orientations with self-efficacy, anxiety, and achievement, doi: 10.1080/09639284.2015.1036892, Accounting Education: an international journal, 24(2), 152-174 (2015)

Dweck, C.S., Motivational processes affecting learning, doi: http://dx.doi.org/10.1037/0003-066X.41.10.1040, American Psychologist, 41(10), 1040-1048 (1986)

Ellis, A. K., D.W., Denton y J.B., Bond, An analysis of research on metacognitive teaching strategies, doi: https://doi.org/10.1016/j.sbspro.2014.01.883, Social and Behavioral Sciences, 116, 4015-4024 (2014)

Gámez, E., J.M. Díaz y otros tres autores, Relaciones entre el Autoconcepto Relacional, la Elección de Metas y la Satisfacción de Necesidades Psicológicas en Estudiantes Universitarios, doi: http://dx.doi. org/10.11144/Javeriana.UPSY13-4.rarm, Universitas Psychologica, 13(4), 1289-1303 (2014)

Hayamizu, T. y B. Weiner, A Test Dweck's Model of Achievement Goals as Related to Perceptions of Ability, doi: 10.1080/00220973.1991.10806562, Journal of Experimental Education, 59(3), 226-234 (1991)

Hayamizu, T., A. Ito y K. Yohiazaki, Cognitive Motivacional Process Mediated by Achievement Goal Tendencies, Japonese Psychological Research, ISSN: 1468-5884, 31, 179-189 (1989)

Honicke, T. y J. Broadbent, The Influence of Academic Self-efficacy on Academic Performance: A Systematic Review, doi.org/10.1016/j.edurev.2015.11.002, Educational Research Review, 17, 63-84 (2016)

Inglés, C.J., D. Aparisi y otros tres autores, Relación entre Tipos Sociométricos y Metas Académicas en una Muestra de Estudiantes Españoles de Educación Secundaria, doi: https://doi.org/10.11144/Javeriana.upsy15-1.rsma, Universitas Psychologica, 15(1), 233-244 (2016)

Komarraju, M. y D. Nadler, Self-efficacy and Academic Achievement: Why Do Implicit Beliefs, Goals, and Effort Regulation Matter?, doi: org/10.1016/j.lindif.2013.01.005, Learning and individual differences, 25, 67-72 (2013)

Krumrei-Mancuso, E.J., F.B. Newton y otros dos autores, Psychosocial Factors Predicting First-year College Student Success, doi: 10.1353/csd.2013.0034, Journal of College Student Development, 54(3), 247-266 (2013)

Lehmann, T., I. Hähnlein y D. Ifenthaler, Cognitive, mecognitive and motivational perspectives on preflection in selfregulated online learning, doi: http://dx.doi.org/10.1016/j.chb.2013.07.051, Computers in Human Behavior, 32, 313-323 (2014)

Linnenbrink, E.A. y P.R. Pintrich, The role of self-efficacy beliefs in student engagement and learning in the classroom, doi: 10.1080/10573560390143076, Reading \& Writing Quarterly, 19, 119-137 (2003)

Linnenbrink, E.A., The dilemma of performance-approach goals: The use of multiple goal contexts to promote students' motivation and learning, doi: 10.1037/0022-0663.97.2.197, Journal of Educational Psychology, 97(2), 197-213 (2005)

Mega, C., L. Ronconi y R. De Beni, What Makes a Good Student? How Emotions, Self-regulated Learning, and Motivation Contribute to Academic Achievement, doi: 10.1037/a0033546, Journal of Educational Psychology, 106(1), 121-131 (2014)

Navas, L., C. González y G. Torregrosa, Metas de Aprendizaje: Un Análisis Transversal de las Estructuras Factoriales que presentan, Revista de Psicología General y Aplicada, ISSN: 0373-2002, 55(4), 553-564 (2002) 
Nunnally, J.C., y I.H. Bernstein, Psychometric Theory, (3ª Ed.), McGraw-Hill, Inc, New York (1994)

Panadero, E., A Review of Self-regulated Learning: Six Models and Four Directions for Research, doi: 10.3389/fpsyg.2017.00422, Frontiers in Psychology, 8, 1-28 (2017)

Pajares, F., Self-efficacy beliefs, motivation, and achievement in writing: a review of the literature, doi: 10.1080/10573560390143085, Reading and Writing Quarterly, 19(2), 139-158 (2003)

Panadero, E. y J.A. Alonso-Tapia, ¿Cómo autorregulan nuestros alumnos? Revisión del modelo cíclico de Zimmerman sobre autorregulación del aprendizaje, doi: http://dx.doi.org/10.6018/analesps.30.2.167221, Anales de Psicología, 30(2), 450-462 (2014)

Panadero, E., A. Jönsson y J. Botella, Effects of self-assessment on self-regulated learning and self-efficacy: Four metaanalyses, doi: 10.1016/j.edurev.2017.08.004, Educational Research Review, 22, 74-98 (2017)

Pérez-Villalobos, M.V., A. Díaz y otros tres autores, Escala de Metas de Estudio para Estudiantes Universitarios, Revista Interamericana de Psicología, 43(3), 449-455 (2009)

Pintrich, P.R., Multiple goals, multiple pathways: The role of goal orientation in learning and achievement, doi: 10.1037/0022-0663.92.3.544, Journal of Educational Psychology, 92(3), 544-555 (2000)

Pool-Cibrian, W.J. y J.I. Martínez-Guerrero, Autoeficacia y Uso de Estrategias para el Aprendizaje Autorregulado en Estudiantes Universitarios, Revista Electrónica de Investigación Educativa, ISSN:1607-4041, 15(3), 21-37 (2013)

Pulkka A.T. y M. Niemivirta, Adult students'achievement goal orientations and evaluations of the learning enviroment: a person-centred longitudinal analysis, doi: 10.1080/13803611.2013.767741, Educational Research and Evaluation, 19(4), 297-322 (2013)

Rosário, P., R. Mourao y otros cuatro autores, Eficacia de un Programa Instruccional para la Mejora de Procesos y Estrategias de Aprendizaje en la Enseñanza Superior, Psicothema, ISSN: 0214-9915, 19(3), $422-427$ (2007)

Rosário, P., J.C. Núñez y otros nueve autores, Transcultural Analysis of the Effectiveness of a Program to Promote Selfregulated Learning in Mozambique, Chile, Portugal, and Spain, doi.org/10.1080/07294360.2014.935932, Higher Education Research y Development, 34(1), 173-187 (2015)

Roth, A., S. Ogrín, S. y B. Schmitz, Assessing Self-regulated Learning in Higher Education: A Systematic Literature Review of Self-report Instrument, doi: 10.1007/s11092-015-9229-2, Educational Assessment, Evaluation and Accountability, 28(3), 225-250 (2016)

Senko C., C.S. Hulleman y J.M. Harackiewicz, Achievement goal theory at the crossroads: Old controversies, current challenges, and new directions, doi: 10.1080/00461520.2011.538646, Educational Psychologist, 46(1), 26-47 (2011)

Sitzmann, T., y K. Ely, A Meta-analysis of Self-regulated Learning in Work-related Training and Educational Attainment: What we know and where we need to go, doi: 10.1037/a0022777, Psychological Bulletin, 137(3), 421-442 (2011)

Taub, M., R. Azevedo y otros dos autores, Can the use of cognitive and metacognitive self-regulated learning strategies be predicted by learners' levels of prior knowledge in hypermedia-learning environments?, doi: http://dx.doi.org/10.1016/j.chb.2014.07.018, Computers in Human Behavior, 39, 356-367 (2014)

Torrano, F. y M. Soria, Diferencias de género y aprendizaje autorregulado: el efecto del rendimiento académico previo, doi: http://dx.doi.org/10.5209/RCED.51096, Revista Complutense de Educación, 28(4), 1027-1042 (2017)

Usher E.L. y F. Pajares, Sources of self-efficacy in school: Critical review of the literature and future directions, doi: 10.3102/0034654308321456, Review of Educational Research, 78(4), 751-796 (2008)

Villavicencio, F.T. y A.B.I. Bernardo, Negative emotions moderate the relationship between self-efficacy and achievement of Filipino students, doi: 10.1007/s12646-013-0193-y, Psychological Studies, 58(3), 225-232 (2013)

Valle, A., R.G. Cabanach y otros tres autores, Metas académicas, estrategias cognitivas y estrategias de autorregulación del estudio, Psicothema, ISSN 0214-9915, 18(2), 165-170 (2006)

Valle, A., B. Regueiro y otros cinco autores, Perfiles Motivacionales como combinación de Expectativas de Autoeficacia y Metas Académicas en Estudiantes Universitarios, doi:10.1016/j.ejeps.2015.10.001, European Journal of Education and Psychology, 8(1), 1-8 (2015)

Weiser, D.A. y H.R. Riggio, Family background and academic achievement: does self-efficacy mediate outcomes?, doi: 10.1007/s11218-010-9115-1, Social Psychology of Education, 13(3), 367-383 (2010)

Wolters, C.A., Advancing achievement goal theory: Using goal estructures and goal orientations to predict students'motivation, cognition, and achievement, doi: 10.1037/0022-0663.96.2.236, Journal of Educational Psychology, 96(2), 236-250 (2004)

Yurt, E., The predictive power of self-efficacy sources for mathematics achievement, doi: 10.15390/EB.2014.3443, Education and Science, 39(176), 159-169 (2014)

Zimmerman, B.J., From Cognitive Modeling to Self-regulation: A Social Cognitive carreer path, doi.org/10.1080/00461520.2013.794676, Educational Psychologist, 48(3), 135-147 (2013) 
